Trauma Berufskrankh 2009. 11[Suppl 1]:78-84 DOI 10.1007/s10039-008-1406-9

Online publiziert: 31. Mai 2008

(c) Springer Medizin Verlag 2008

\author{
J. Madert $\cdot$ C. Eggers \\ Chirurgisch-Traumatologisches Zentrum, AK St. Georg, Hamburg
}

\title{
Fallstricke in der Wirbelsäulendiagnostik
}

vorne und seitlich an den Kopf. Sie sind sekundäre Stabilisatoren und reißen daher auch sekundär. Die Gelenkflächen stehen sagittal, sind konvex und haben lockere Bänder. Hier sind nur eine geringe Flexion/Extension und Seitneigung möglich, allerdings besteht eine große Rotationsmöglichkeit von insgesamt $80^{\circ}$. Auch in diesem Bereich ist der Spinalkanal relativ weit.

\section{Halswirbelsäule}

\section{Anatomie}

Zwischen dem Hinterhaupt und dem HWK1 findet man nur wenige Ligamente, die Gelenke haben eine flache muldenförmige Form. In diesem Bereich ist eine kombinierte Flexion/Extension von etwa $25^{\circ} \mathrm{möglich}$, die Seitneigung ist gering, die axiale Rotation beträgt maximal $12^{\circ}$, der Spinalkanal ist sehr weit.

Während die Wirbel von $\mathrm{HWK}_{3}-$ $\mathrm{LWK}_{5}$ die gleiche anatomische Grundstruktur aufweisen, zeigen der HWK1 und -2 Besonderheiten, u. a. fehlt ihnen der Wirbelkörper. Sie bilden 2 aufeinandergelegte Ringe, wobei von dem unteren ein zahnartiger Fortsatz (der Dens) den Drehzapfen für den HWK1 darstellt. Der Dens ist nach dorsal durch ein quer verlaufendes Band (Lig. transversum) gesichert. Die Ligg. alaria ziehen von der Densspitze zum Bogen des HWK1 nach

\section{Abkürzungen}

\begin{tabular}{|ll}
\hline BWK & Brustwirbelkörper \\
\hline BWS & Brustwirbelsäule \\
\hline HWK & Halswirbelkörper \\
\hline$H W S$ & Halswirbelsäule \\
\hline LWK & Lendenwirbelkörper \\
\hline LWS & Lendenwirbelsäule \\
\hline WS & Wirbelsäule \\
\hline
\end{tabular}

\section{Verletzungen}

Obere HWS. Traumen der oberen HWS sind selten und machen nur $20 \%$ der gesamten HWS-Verletzungen aus. Durch ihre enge Beziehung zur A. vertebralis und der Medulla oblongata können sie u. U. katastrophale Folgen nach sich ziehen und verlaufen häufig tödlich, wenn es um den Bereich Co/C1 geht. Radiologisch sind sie wegen knöcherner Überlagerungen und feinen anatomischen Strukturen schwierig zu diagnostizieren. Häufig finden sich weitere Wirbelsäulenverletzungen.

Im Bereich des $\mathrm{HWK}_{1} /-2$ ist besonders die Densfraktur hervorzuheben. Sie stellt bei Patienten, die älter als 70 Jahre sind, die häufigste Halswirbelsäulenverletzung dar [11].

Untere HWS. In diesem Bereich werden nahezu die gesamte Extension/Flexion (maximal $\left.\mathrm{C}_{5} / \mathrm{C} 6\right)$ sowie die Seitneigung (maximal $\mathrm{C}_{4} / \mathrm{C}_{5}$ ) durchgeführt. Der Spinalkanal ist mäßig weit. Die untere HWS geht als flexibelster Teil der Wirbelsäule in die starre Brustwirbelsäule über, die auch einen Scheitelpunkt der s-förmigen Konfiguration darstellt.

In der unteren HWS sind $80 \%$ der Halswirbelsäulenverletzungen lokalisiert. Der am häufigsten gebrochene Wirbel- körper ist der $\mathrm{HWK}_{5}$, das am häufigsten befallene Segment ist $\mathrm{HWK}_{5} / 6$ [4].

Zusammenfassend muss festgehalten werden, dass man bei Patienten mit HWSVerletzung ein besonderes Augenmerk auf den Bereich $\mathrm{HWK}_{5}-\mathrm{HWK}_{7}$ und bei älteren Patienten auf die Dens-axis-Region legen sollte, da dort die häufigsten Verletzungen auftreten.

\section{Brustwirbelsäule}

\section{Anatomie}

In der BWS finden sich eine deutliche $\mathrm{Zu}$ nahme der Steifigkeit (2,5- bis 4-fach) und eine Kyphosestellung der Wirbelsäule [1, 13]. Die Gelenkstellung ist eher frontal, daher ist hier die Rotation möglich. Das hintere Längsband ist in diesem Bereich am ausgeprägtesten, da es auf Zug beansprucht wird. Zwischen BWK4 und -8 finden sich die schmalsten Pedikel und der engste Spinalkanal der gesamten Wirbelsäule [2].

Im Bereich des thorakolumbalen Übergangs gelten die gleichen biomechanischen Bedingungen wie beim zervikothorakalen Übergang (Scheitelpunkt der S-Konfiguration, Übergang des starren zum flexiblen Bereich).

\section{Verletzungen}

Der thorakolumbale Übergang ist am häufigsten betroffen [6].

Die erhöhte Steifigkeit bedingt, dass die Betroffenen einen hohen Anteil an Begleitverletzungen aufweisen. So fanden sich nach Blauth et al. [3] bei etwa $40 \%$ der Patienten zusätzliche Thoraxverletzungen:

\section{- Rippenserienfrakturen traten sehr} häufig auf. 
- Die 1. und 2. Rippe waren in 10\% betroffen.

- In $36 \%$ der Fälle fand sich ein Hämatopneumothorax.

- In fast $69 \%$ lag eine Mediastinalverbreiterung vor.

- Zusätzlich Klavikula- und Skapulafrakturen zeigten sich in $2-5 \%$.

- $42 \%$ der Patienten hatten eine zusätzliche Kopfverletzung.

Frakturen der 1. und 2. Rippe sind ein Zeichen einer deutlich höheren Gewalteinwirkung und immer als Hinweis auf eine evtl. zusätzliche Wirbelsäulenverletzung ernst zu nehmen. Das bedeutet im Umkehrschluss, bei Frakturen der 1. und 2. Rippe ist eine zusätzliche WS-Fraktur auf jeden Fall auszuschließen.

\section{Lendenwirbelsäule}

Durch die sagittale Gelenkstellung kommt es zu einem sprunghaften Anstieg der Rotationssteifigkeit. Hier sind hauptsächlich die Extension und Flexion möglich, der Spinalkanal ist weit, im unteren Anteil findet sich die Cauda equina.

Im Bereich der LWS fehlt die stabilisierende Wirkung des Thorax, der 1. Lendenwirbelköper stellt wie oben erwähnt den Scheitelpunkt dar, sodass er der am häufigsten betroffene und verletzte Wirbelkörper überhaupt ist [6].

\section{Os sacrum}

In diesem Bereich fehlen die Zwischenwirbelgelenke. Das Os sacrum bildet den dorsalen Teil des Beckenrings und wirkt wie ein Schlussstein in einem Gewölbe, obwohl er es biomechanisch nicht ist.

In unmittelbarer Nachbarschaft befindet sich ein großer Gefäßplexus, der immerhin für $3 \%$ der hämorrhagischen Schocks bei Beckenverletzungen verantwortlich ist [9]. Deren Detektion sollte im Schockraumalgorithmus enthalten sein.

Dem Os sacrum vorgelagert sind großvolumige Weichteile, die diagnostische Schwierigkeiten bereiten.

Trauma Berufskrankh 2009 · 11[Suppl 1]:78-84 DOI 10.1007/s10039-008-1406-9

○) Springer Medizin Verlag 2008

\section{J. Madert $\cdot$ C. Eggers \\ Fallstricke in der Wirbelsäulendiagnostik}

\section{Zusammenfassung}

Traumen der oberen Halswirbelsäule (HWS) sind selten. Häufig befallene Regionen sind der Dens axis bei Patienten $>70$ Jahre, der HWK5 (Halswirbelkörper 5), das Segment HWK5/6, der BWK12 (Brustwirbelkörper 12) und der LWK1 (Lendenwirbelkörper 1). Die Diagnostik beinhaltet die gründliche klinische Untersuchung der gesamten Wirbelsäule inklusive Os sacrum, um nicht die klassischen Verletzungshöhen (zervikothorakal, thorakolumbar, Os sacrum) zu übersehen. Bei Standard- und Funktionsröntgenaufnahmen müssen alle HWK abgebildet sein. Bei Morbus Bechterew wird das Verletzungsausmaß meist unterschätzt, bei vorbestehender degenerativer Veränderung lassen Funktionsaufnahmen oft keine deutliche Verschiebung der Wirbelkörper erkennen. Wegen der

\section{Pitfalls of spinal injury diagnosis}

\section{Abstract}

Injuries of the upper cervical spine are rare. The most commonly affected regions are the dens axis in patients older than 70 years, the 5 th cervical body, the spinal segment $C 5 / 6$, the 12th thoracic body, and the 1st lumbar body. Diagnosis involves thorough physical examination of the entire spinal column, including the os sacrum, in order not to miss the classical injury locations (cervicothoracic, thoracolumbar, os sacrum). Standard x-ray and functional images must show all cervical bodies. The presence of ankylosing spondylitis often leads to underestimation of the extent of injury; the clinician must pay close attention to degenerative spinal alterations because functional images may show only minimal shifting of the vertebral bodies. Because schlechten Sicht im Brustwirbelsäulenbereich ist die Indikation zum CT großzügig stellen, Begleitverletzungen müssen abgeklärt werden. Allgemeine Ursachen der Fallstricke sind mangelnde pathophysiologische Kenntnisse (z. B. häufig betroffene Regionen), das Ignorieren anamnestischer Hinweise und neurologischer Befunde (das „,komische" Gefühl im kleinen Finger als Hinweis auf eine HWSVerletzung) und die oberflächliche Untersuchung (z. B. Untersuchung lediglich der unteren Lendenwirbelsäule bei Fällen von Rückenschmerz).

\section{Schlüsselwörter}

Wirbelsäulendiagnostik · Becken · Fallstricke . Häufigste Verletzungen · Klinische/apparative Untersuchung of poor visibility in the thoracic region, computed tomography should be used generously to clarify thoracic injuries. General diagnostic pitfalls include insufficient pathophysiologic knowledge (such as the most frequently affected regions), ignoring anamnestic and neurological findings (for example, a "strange feeling" in the 5th finger as an indication of cervical spine injury), and only superficial physical examination (for instance, examining only the lower lumbar region in cases of back pain).

\section{Keywords}

Spinal diagnostic $\cdot$ Pelvis $\cdot$ Pitfalls of spinal injuries · Frequency of spinal injuries .

Clinical/radiological diagnosis 


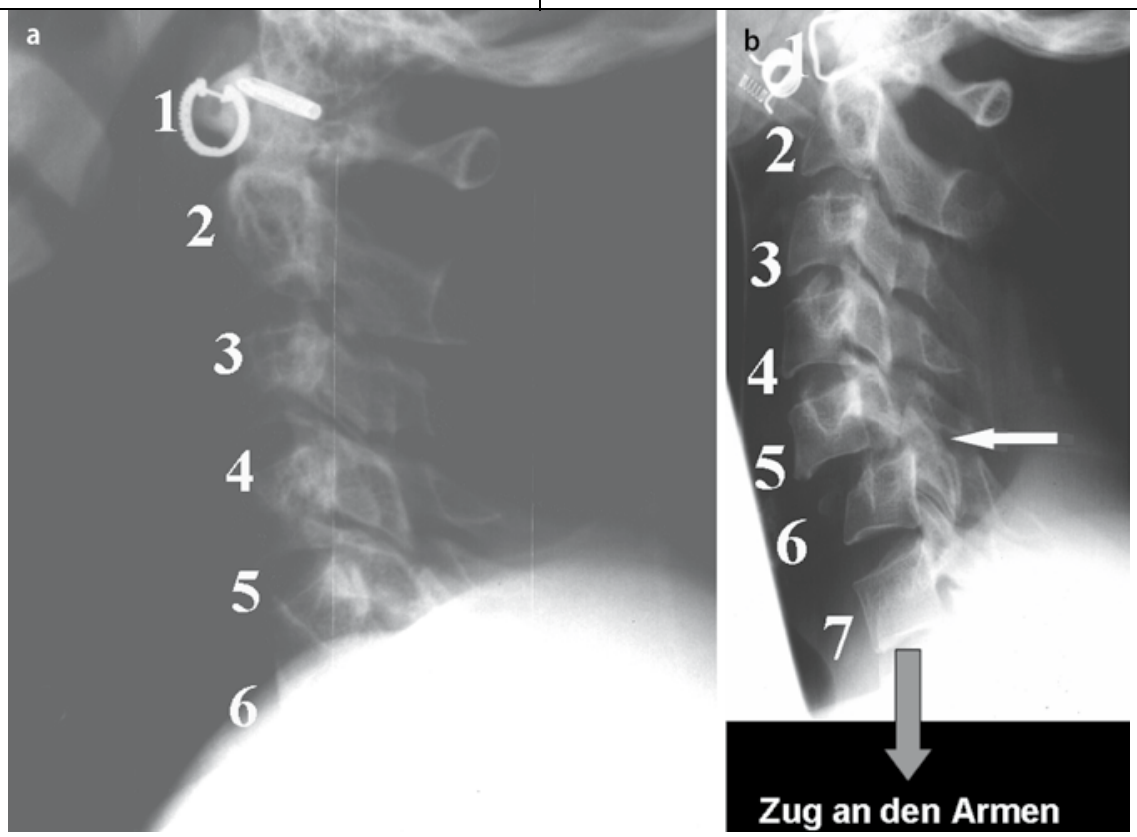

Abb. $1 \Delta$ Röntgenaufnahmen der HWS, a inkorrekte Aufnahme seitlich, b korrekte Aufnahme mit Darstellung aller Wirbelkörper

\section{Allgemeine Diagnostik}

\section{Anamnese}

Die Unfallanamnese gibt Hinweise, so sollten der Sprung in unbekanntes flaches Wasser, stumpfe Rasanztraumen, direkte Verletzungen der Hals- und Nackenregion, Sturz aus größerer Höhe, Rotationstraumen, Mehrfach- sowie Kopfverletzungen Anlass sein, die Wirbelsäule exakt zu untersuchen.

Der 18-jährige junge Mann, der in die Alster springt und mit HWS-Beschwerden in die chirurgische Aufnahme kommt, ist hochverdächtig auf eine diskoligamentäre Instabilität der HWS. Bei demjenigen, der aus dem 3. Stock fällt, ist an eine Fraktur im thorakolumbalen Übergang zu denken, und bei dem 80 Jahre alten Patienten, der vornüber aus seinem Sessel stürzt, muss eine Densfraktur ausgeschlossen werden.

\section{Klinik}

Die klinische Untersuchung ist Weg weisend. So sind lokale Abschürfungen und Kontusionen, Druck- und Klopfschmerz, Beweglichkeit der HWS, Hämatome und neurologische Symptome ernst zu nehmen.

Ein klassischer Fallstrick bei der Eruierung von Verletzungen im Bereich der
Wirbelsäule ist der so genannte Rückenschmerz. Dieser Begriff impliziert Schmerzen im Bereich der unteren LWS. Häufig lassen sich dort sogar Klopfschmerzen auslösen, obwohl die Verletzung evtl. kranialer liegt. So sollte in jedem Fall die gesamte Wirbelsäule klinisch gründlich untersucht werden, damit man die statistisch häufigste Verletzungen (BWK12/LWK1) nicht übersieht.

Bei Patienten mit Os-sacrum- bzw. Beckenverletzungen zeigen sich 2 Altersgipfel. Die eine Gruppe betrifft die 20- bis 25-Jährigen, die andere die 80- bis 85-Jährigen. Aus den beiden Altersgipfeln lässt sich die unfallbedingte Krafteinleitung ableiten. Während in der jungen Gruppe häufig Rasanztraumen die Ursache darstellen, finden sich in der höheren Altersgruppe eher harmlose Ursachen wie der häusliche Sturz. Führend sind in der jungen Gruppe die Männer, in der Altersgruppe die Frauen [5, 9].

Ob nun ein polytraumatisierter Patient oder der Ältere in die chirurgische Aufnahme kommt, abzuklären ist bei dementsprechenden Hinweisen eine Beckenverletzung. Die klinische Untersuchung, d.h. das Auslösen von Schmerzen bei Kompression, Extension und Rotation der Beckenschaufel zeigt leider nach Pehle et al. [8] nur eine Sensibilität von $44 \%$, d. h. in mehr als der Hälfte der Fälle ist klinisch nicht zu eruieren, ob eine dorsale Becken- verletzung vorliegt. Auf der anderen Seite lassen sich bei 98\% der Patienten mit dorsalen Verletzungen bei der Palpation und dem Abklopfen Schmerzen auslösen [7], sodass diese beiden Methoden auf jeden Fall zum Algorithmus der Beckenuntersuchung bei vigilanten Patienten gehören.

\section{Apparative Diagnostik}

Sie bringt die Bestätigung. Zur Verfügung stehen:

- Röntgen,

- Computertomographie und

- Magnetresonanztomographie.

Alle Untersuchungen sind in Abhängigkeit des Vigilanzgrades der Patienten durchzuführen. Ältere Menschen, Kinder, sedierte oder gar intubierte Patienten sind in ihrer Vigilanz deutlich eingeschränkt und erfordern damit eine großzügige apparative Untersuchung. Bei einem polytraumatisierten Patienten, der intubiert und beatmet in den Schockraum transportiert wird, ist diskussionslos ein Traumascan durchzuführen. Auf der anderen Seite ist bei dem jungen vigilanten Motorradfahrer, der schmerzfrei seine HWS in allen Richtungen bewegen kann, keine apparative Diagnostik notwendig.

\section{HWS}

Röntgen nativ. Zur Vervollständigung der Diagnostik werden eine a.-p. und eine seitliche Aufnahme der HWS sowie eine Denszielaufnahme transoral durchgeführt. Lassen sich keine knöchernen Verletzungen nachweisen, muss mittels Funktionsaufnahmen die diskoligamentäre Instabilität ausgeschlossen werden. Aktive Funktionsaufnahmen, wobei der Patient selbst die HWS flektiert und extendiert, dürfen nur bei wachen, verständigen $\mathrm{Pa}$ tienten erstellt werden. So genannte geführte Aufnahmen, bei denen der Arzt den Kopf in die Hand nimmt und die Halswirbelsäule in- und rekliniert, müssen unter ständiger Bildwandlerkontrolle durchgeführt werden, um evtl. Verschiebungen der Wirbelkörper gegeneinander oder ein Klaffen der Facettengelenke sofort zu erkennen.

In Anbetracht der Tatsache, dass der Bereich $\mathrm{HWK}_{5}-\mathrm{HWK}_{7}$ das am häufigs- 

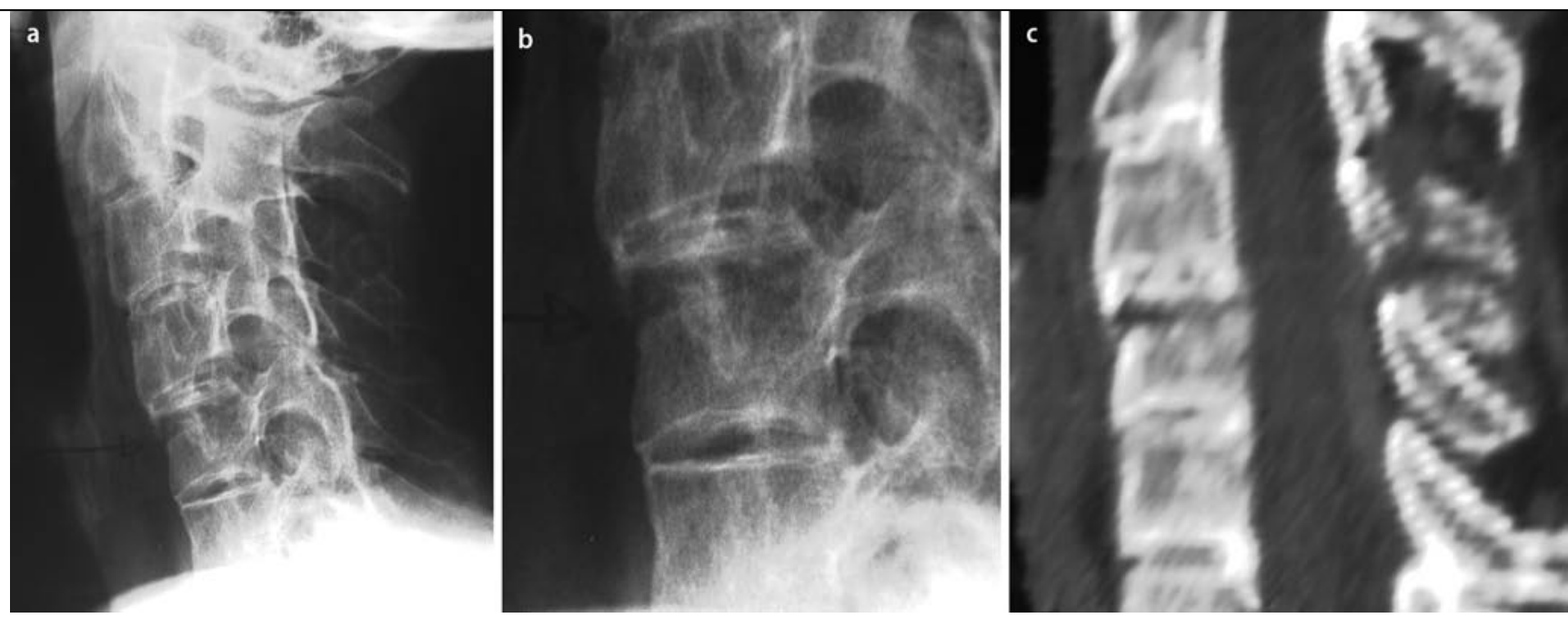

Abb. 2 \ HWS-Fraktur bei Morbus Bechterew, a Röntgen seitlich, b Röntgenausschnitt, c CT mit wahrem Verletzungsausmaß

ten betroffene Areal ist, sollte man bis 7 zählen, d. h. alle Halswirbelkörper müssen auf der Aufnahme zu sehen sein, da ansonsten die Gefahr der übersehenen Verletzung besteht, wie an den Beispielaufnahmen in - Abb. 1 zu sehen ist.

Einen weiteren Fallstrick stellen die Patienten mit Morbus Bechterew dar. Bedingt durch die Ossifikationen der Ligamente und die knöcherne Rarefizierung der Wirbelkörper sind die Röntgenaufnahmen im Kontrast deutlich reduziert, sodass man einzelne anatomische Strukturen nicht gut abgrenzen kann (• Abb. 2). Des Weiteren kommt in Anbetracht der Rigidität der Wirbelsäule hinzu, dass das Verletzungsausmaß meist wesentlich größer ist als man annimmt (• Abb. 2), mit der großen Gefahr der Dislokation, da der Hebelarm zu beiden Seiten der Verletzung sehr lang ist und damit enorme Kräfte auf den Bruch wirken.

Der Weichteilschatten sollte im Bereich $\mathrm{HWK}_{1} / 2$ bis ungefähr $\mathrm{HWK}_{4}$ etwa $4-5 \mathrm{~mm}$ betragen, um dann physiologisch auf 12-16 $\mathrm{mm}$ anzusteigen. So zeigt - Abb. 3 eine unauffällige knöcherne Halswirbelsäule, jedoch findet sich prävertebral ein starker Weichteilschatten in Höhe $\mathrm{HWK}_{2}-\mathrm{HWK}_{4}$, sodass die etwas erweiterte Gelenkstellung zwischen $\mathrm{HWK}_{4}$ und -5 die Aufmerksamkeit auf sich lenkt und weitere Diagnostik, z. B. Funktionsaufnahmen, fordert. Da sich die HWS bei dem in $\bullet$ Abb. 3 dargestellten Fall beim Röntgen in korrekter In-Line-Lagerung befand, lag bei diesem Patienten mit einer diskoligamentären Instabilität keine Luxationsstellung vor.

Bei evtl. durchgeführten Funktionsaufnahmen gilt ebenfalls, dass im Prinzip alle Halswirbelkörper abgebildet sein und eine echte In- und Reklination vorliegen müssen. Während bei jüngeren Patienten die Stufenbildung im Fall einer Instabilität häufig nicht zu übersehen ist, stellt die Instabilität bei Patienten mit einer stark degenerativ veränderten HWS ein Problem dar (• Abb. 4).

Bei einer Verschiebung auch nur um einige Millimeter bei klinischem Verdacht auf eine Instabilität ist eine weiterführende Diagnostik anzustreben. In solchen Fällen ist eine MRT hilfreich, da mit ihr eine frische Verletzung aufgrund der Ödembildung detektiert werden kann.

Computertomographie. Sie ist das eigentliche Diagnostikum zur Beurteilung von Frakturen. Früher extra angefordert, heute in der Regel im Standardprogramm sollten koronare, sagittale und axiale Schichten erstellt werden.

Das CT bietet die Möglichkeit der Beurteilung der Frakturausdehnung, des Verlaufs der Instabilitätslinie, des Dislokationsgrades von Hinterkantenfragmenten, der Luxationen von Facettengelenken und Wirbelkörpern, der Weite des Spinalkanals sowie der Struktur der Spongiosa.

Eine rein axiale Schichtung ist nicht ausreichend. $\mathrm{Zu}$ verlangen sind eine Rekonstruktion bzw. eine sagittale Aufnahme, da nur in ihnen Subluxationen deutlich dargestellt werden können (• Abb. 5).

Magnetresonanztomographie. Sie ermöglicht die Beurteilung der Weichteilstrukturen wie Ligamente und Bandscheiben. Mit der MRT können raumfordernde Hämatome, Tumoren, Traumen und Entzündungen nachgewiesen werden. Bei Patienten mit Osteoporose hilft sie, den Fallstrick des vermeintlich alten Bruchs zu umgehen. Durch das im MRT sichtbare Ödem lassen sich alte von frischen Frakturen unterscheiden.

Resümee. Wichtig ist immer, das Röntgenbild kritisch $\mathrm{zu}$ analysieren und $\mathrm{zu}$ werten. Es müssen alle Halswirbelkörper abgebildet sein.

Besondere Aufmerksamkeit sollte dem Morbus Bechterew, bei welchem das Verletzungsmaß meist unterschätzt wird, sowie einer vorbestehenden degenerativen Veränderung zukommen, da Funktionsaufnahmen häufig keine deutliche Verschiebung der Wirbelkörper erkennen lassen. Der Weichteilschatten darf nicht vergessen werden, da bei In-Line-Lagerung der Wirbelsäule häufig korrekte anatomische Verhältnisse vorgetäuscht werden.

\section{BWS/LWS}

Röntgen nativ. Bei Verletzungen im Bereich der BWS und LWS werden in der Regel Röntgenbilder in 2 Ebenen angefertigt, wobei der thorakolumbale Über- 


\section{Der vermeintlich einfache Fall}

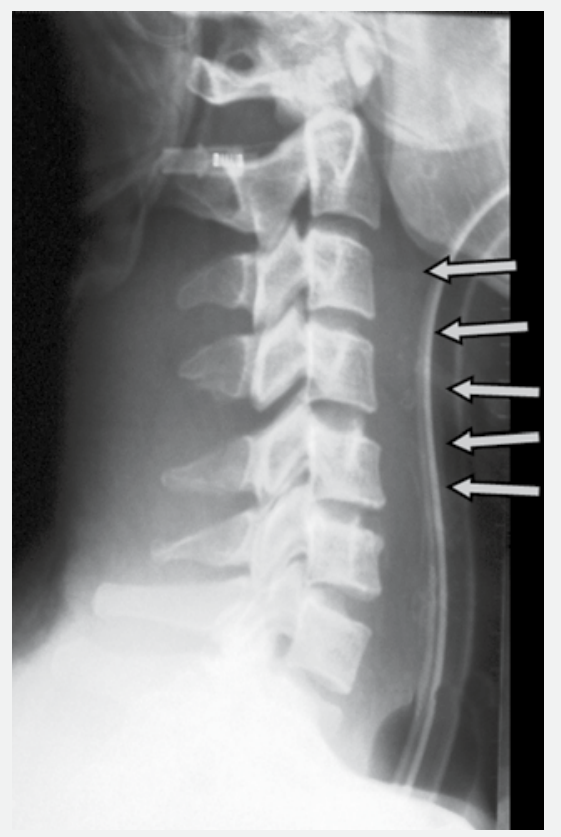

Abb. $3 \Delta$ Breiter Weichteilschatten, HWK2HWK4

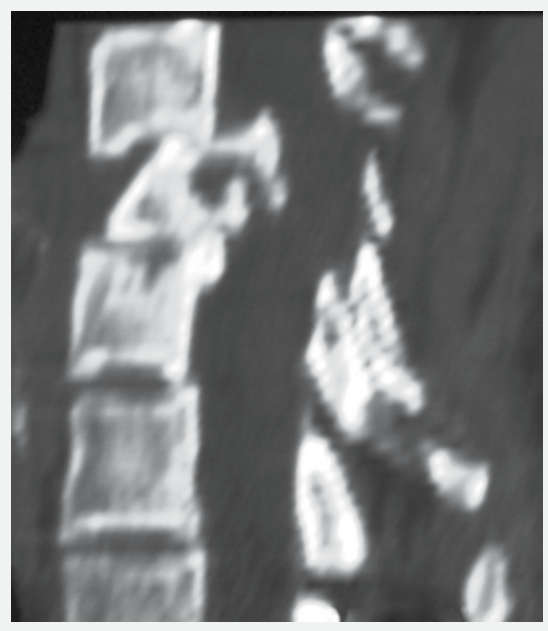

Abb. $5 \Delta \mathrm{CT}$, seitliche Rekonstruktion der HWS, Subluxation

gang zusätzlich geröntgt werden sollte. Wegen Weichteilüberlagerung im Bereich der Brustwirbel besteht dort eine schlechte Sicht.

Die a.-p. Aufnahme dient zur Beurteilung der Abweichung der Dornfortsätze (Rotation/Distraktion), Verbreiterung des Abstands der Pedikelfiguren bzw. des Wirbelkörpers, der Querfortsätze und einer evtl. Translation der gesamten Wirbelsäule (『 Abb. 6a).

In der seitlichen Aufnahme kann man die Höhe der Wirbelkörpervorder- und -

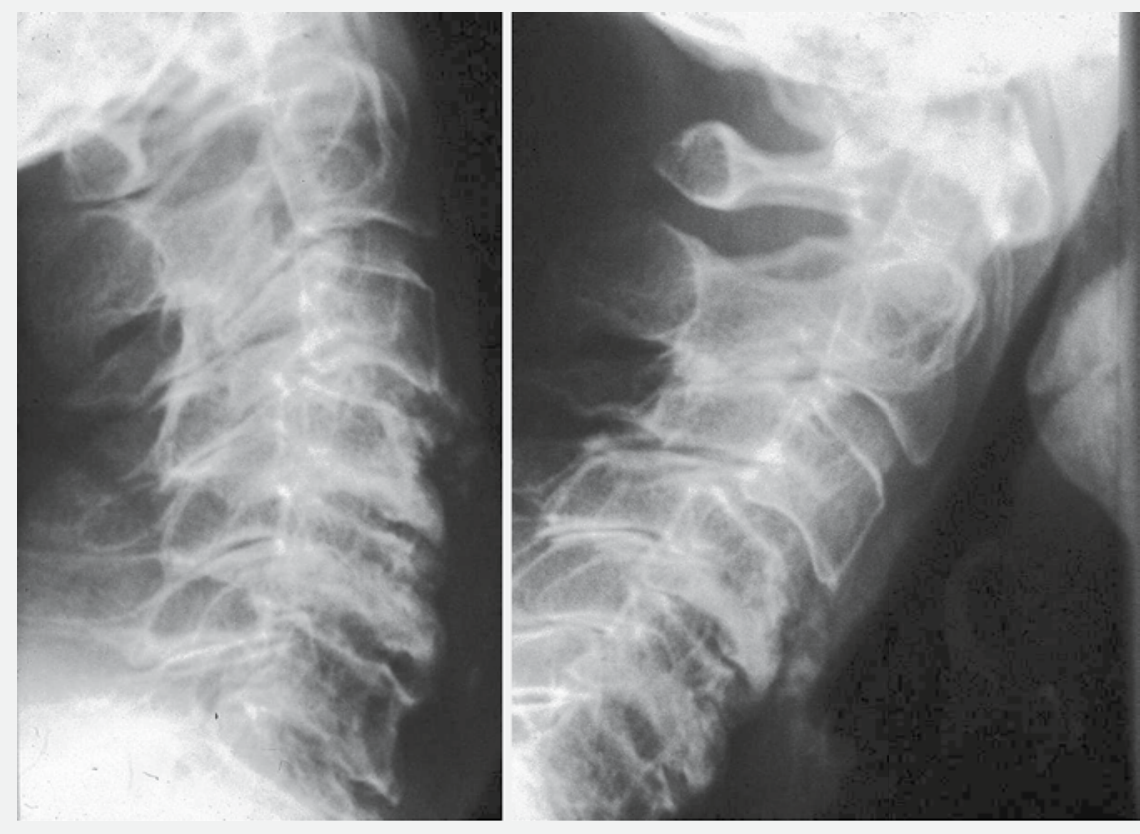

Abb. $4 \Delta$ Funktionsaufnahmen bei degenerativ veränderter HWS
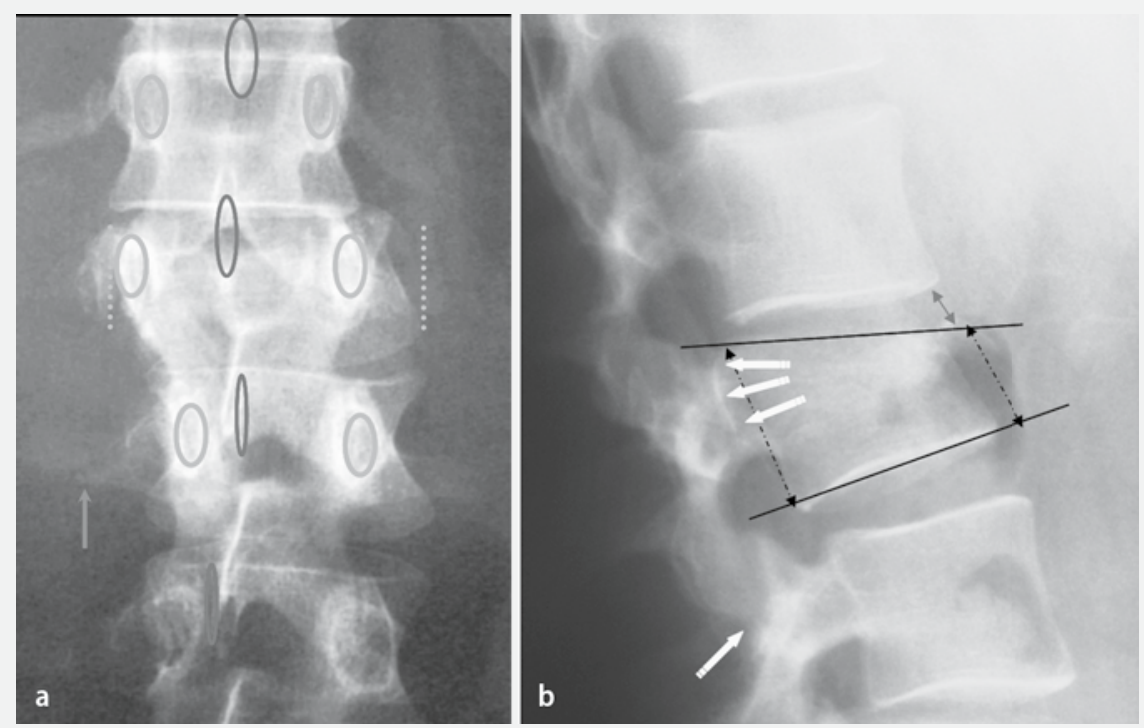

Abb. $6 \Delta$ Beurteilungskriterien beim Röntgen der Wirbelsäule, a a.-p., b seitlich

hinterkante, den Verlauf der Hinterkante, die Höhe des Bandscheibenraums, die Stellung der Gelenkfacetten und die Instabilitätslinie erkennen ( $\mathbf{\square}$ Abb. 6b).

Ein Fallstrick stellt die Röntgenanordnung dar. Ähnlich wie in der Anamnese der Rückenschmerz eine untere LWSVerletzung impliziert, wird bei Auslösung von Klopfschmerzen im Bereich des thorakolumbalen Übergangs gerne „Rö. BWS und LWS in 2 Ebenen " angeordnet mit der Folge, dass der Bruch evtl. am Bildrand zu liegen kommt und dann übersehen wird (- Abb. 7). Besteht der Verdacht auf eine Verletzung des thorakolumbalen Übergangs, sollte dies auch explizit durch „Röntgen thorakolumbaler Übergang in 2 Ebenen" geklärt werden.

Computertomographie. Wie bei der HWS ist das CT das eigentliche Diagnostikum. Hier können der Frakturverlauf und das Ausmaß dislozierter Hinterkantenfragmente, Facetten- und Wirbelkörperluxationen, die Weite des Spinalkanals, der Pedikeldurchmesser, die Dornfortsät- 


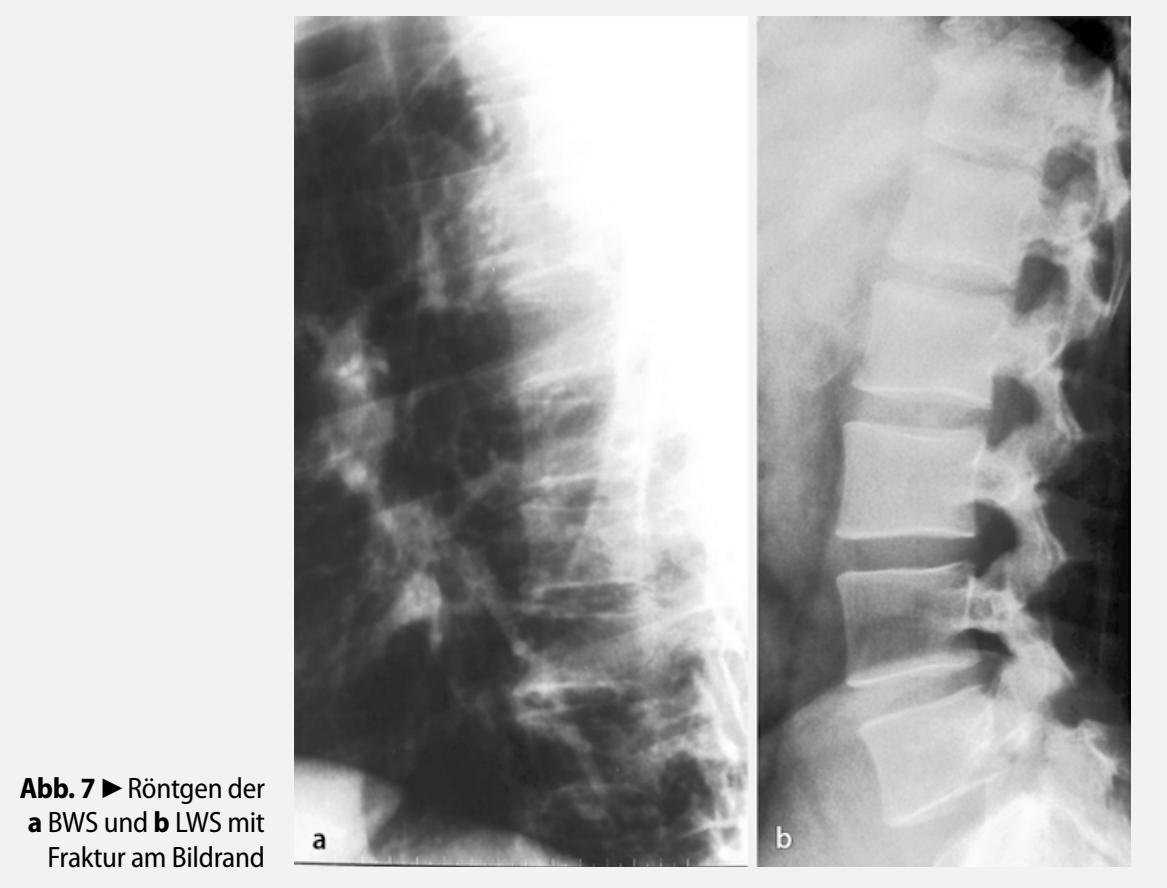

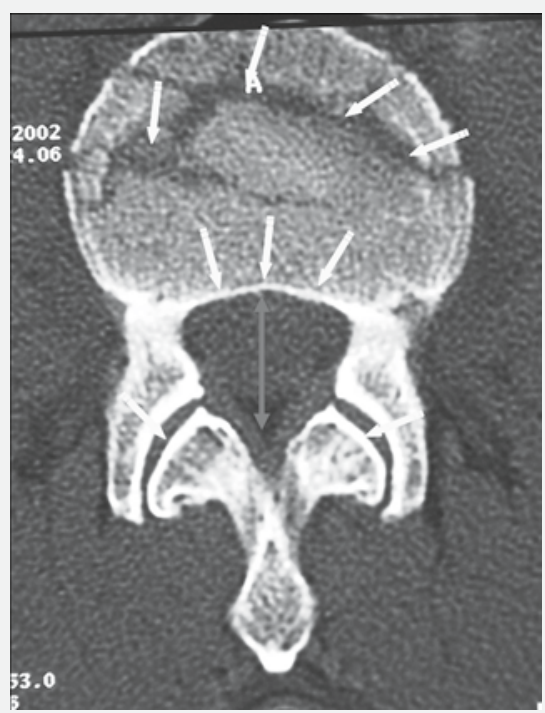

Abb. $8 \triangle$ Beurteilungskriterien CT axial

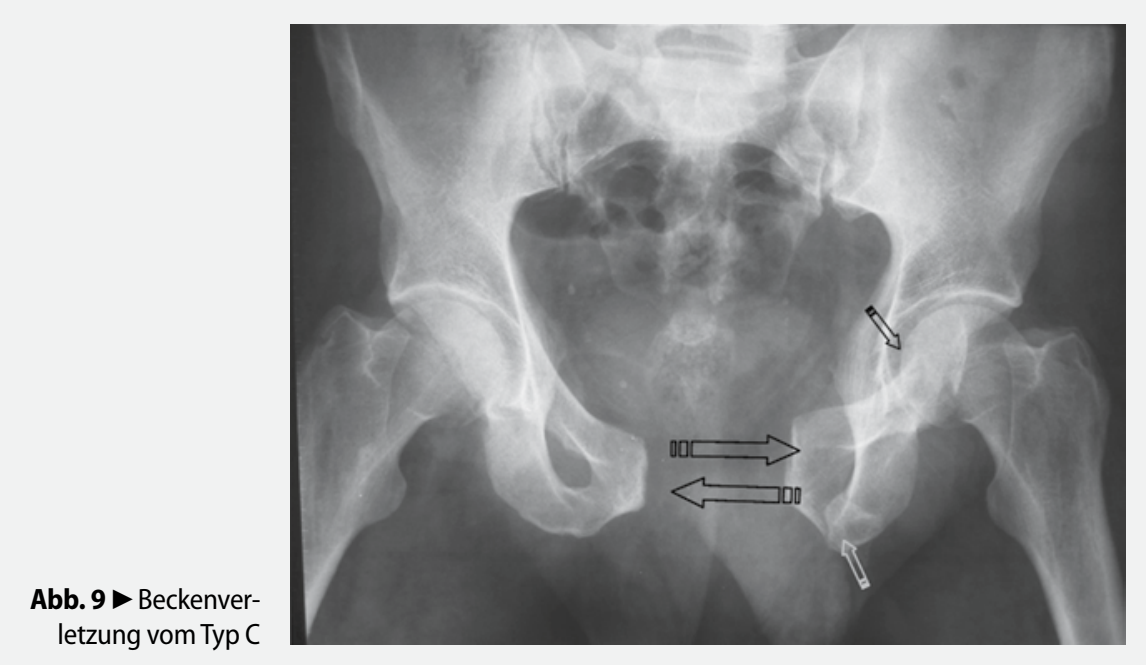

Abb. $10>0$ Os-sacrum-Fraktur, Unterbrechung der Foramenlinien, a Röntgen, b CT
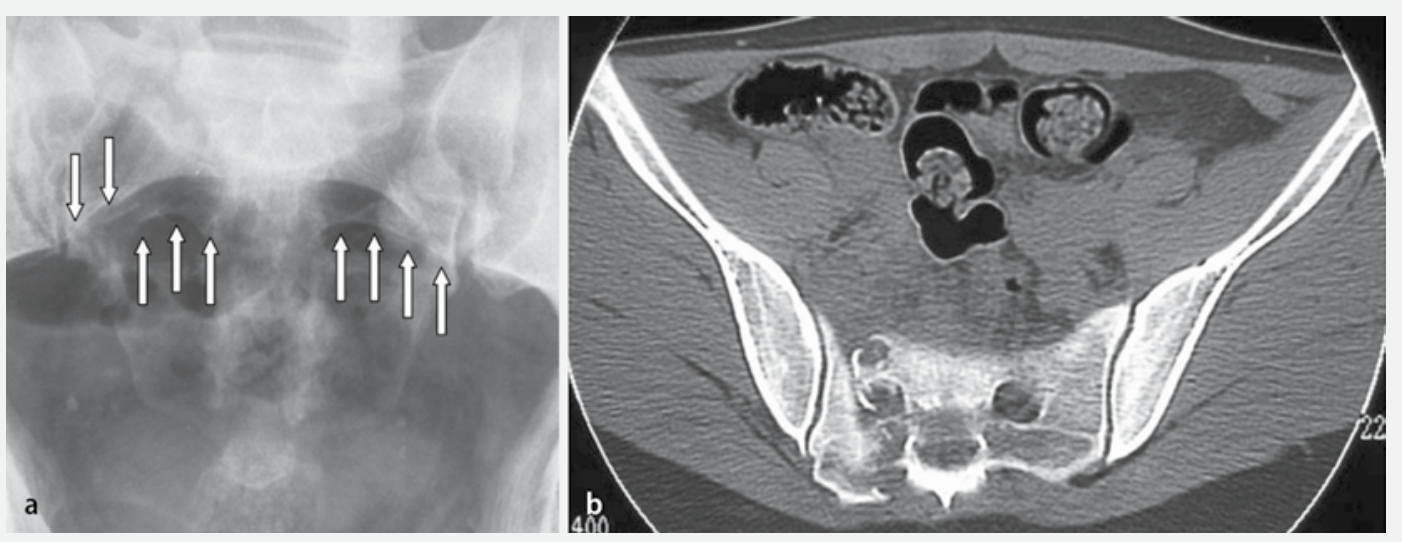
ze und die Struktur der Spongiosa beurteilt werden ( $\bullet$ Abb. 8). Auch hier sind in der heutigen Zeit eine koronare, sagittale und axiale Schichtführung selbstverständlich.

Magnetresonanztomographie. Hier gilt dasselbe wie im Bereich der HWS.

Myelographie/Myelo-CT. Sie sind dann sinnvoll, wenn Metall Artefakte produziert oder ein MRT nicht möglich ist, z. B. aufgrund eines Schrittmachers.

Resümee. Fasst man den BWS-/LWSBereich zusammen, gilt auch hier, dass man die Röntgenbilder kritisch analysieren und werten sollte. Auch hier stellt der Morbus Bechterew eine Klippe dar.

Man muss den Ort des klinischen Befunds röntgen und sich nicht auf Standardanordnungen zurückziehen.

Wegen der schlechten Sicht im BWSBereich sollten die Indikation zum Computertomogramm großzügig gestellt und vor allen Dingen Begleitverletzungen abgeklärt werden.

\section{Becken}

Röntgen nativ. Bei Verdacht auf eine Beckenverletzung wird in erster Linie eine Beckenübersichtsaufnahme durchgeführt. Auf dieser sind Beckenschaufelund vordere Beckenringfrakturen in der Regel gut auszumachen (• Abb. 9). Problem bleibt das Os sacrum. Man stößt auf eine ähnliche Problematik wie bei der klinischen Untersuchung. Auf den Nativbildern lassen sich nur etwa 50\% der knöchernen oder ligamentären dorsalen Verletzungen erkennen [10, 12].

Die genaue Betrachtung der feinen Linien der Foramina des Os sacrum gibt häufig bereits Hinweise. Eine Unterbrechung der Linien sollte die Indikation zu einer weiterführenden Diagnostik sein (- Abb. 10a), ebenso wie die Abrissfraktur des LWK5-Querfortsatzes, da von diesem ein Ligament zur Beckenschaufel zieht.

Zum Standard der Diagnostik gehören eine Inlet- und eine Outletaufnahme des Beckens. Bei den heutigen jedoch überall zur Verfügung stehenden, hoch auflö- senden Computertomographen relativiert sich die Indikation.

Computertomographie. Das eigentliche diagnostische Kriterium für das Becken bzw. das Os sacrum ist das Computertomogramm. Es zeigt den exakten Frakturverlauf in den verschiedenen Ebenen sowie den Dislokationsgrad (• Abb. 10b).

Resümee. Fasst man zusammen, ist nur bei vigilanten Patienten klinisch die dorsale Verletzung des Beckenrings, also des Os sacrum, zu eruieren, ansonsten sollte die Indikation zum CT großzügig gestellt werden.

\section{Fazit}

Allgemeine Ursachen der Diagnostikfallstricke sind:

- mangelnde pathophysiologische Kenntnisse (z. B. häufig betroffene Regionen der Wirbelsäule),

- das Ignorieren anamnestischer Hinweise,

- die Nichtbeachtung neurologischer Befunde (das „,komische“ Gefühl im kleinen Finger als Hinweis auf eine HWS-Verletzung),

- die oberflächliche Untersuchung (z. B. Rückenschmerz: Untersuchung lediglich der unteren LWS, Röntgen ohne Standardeinstellung).

\section{Hauptursache scheint uns jedoch die} menschliche Schwäche zu sein, die uns dazu verführt, es an Systematik fehlen zu lassen. Allzu häufig betrachten, untersuchen und diagnostizieren wir gerne nur das, was wir erwarten, anstatt strukturiert, konsequent algorithmisch vorzugehen.

\section{Korrespondenzadresse}

\section{Dr. J. Madert}

Chirurgisch-Traumatologisches Zentrum, AK St. Georg, Lohmühlenstraße 5, 20099 Hamburg j.madert@asklepios.com

Interessenkonflikt. Der korrespondierende Autor gibt an, dass kein Interessenkonflikt besteht.

\section{Literatur}

1. Andriacchi TP, Schultz AB, Belytschko TB et al. (1974) A model of studies of mechanical interaction between the humane spine and rib cage. J Biomech 7: 497-508

2. Anetzberger H, Friedl HP, Putz R et al. (1997) Wirbelsäule. In: Platzer W, Trentz O (Hrsg) Posttraumatische Defektsanierung und Infektsanierung, Schädel, Wirbelsäule, Becken. Chirurgische Operationslehre, Bd 8. Thieme, Stuttgart New York, S 300-409

3. Blauth M, Knop C, Bastian L (1998) Brust- und Lendenwirbelsäule. In: Tscherne H, Blauth M (Hrsg) Tscherne Unfallchirurgie - Wirbelsäule. Springer, Berlin Heidelberg New York, S 24, 241, 276, 303

4. Bohlmann HH (1979) Acute fractures and dislocation of the cervical spine. An analysis of 300 hospitalized patients and review of literature. J Bone Joint Surg 61: 1119-1142

5. Culemann U, Tosounidas G, Reilmann $\mathrm{H}$ et al. (2004) Beckenringverletzungen. Unfallchirurg 12: 1169-1181

6. Knop C, Blauth M, Buhren V et al. (1990) Operative treatment of fractures and dislocations of thoracolumbar spine - 1: Epidemiology. Unfallchirurg 102: 924-935

7. McCormick JP, Morgan SJ, Smith WR (2003) Clinical effectiveness of the physical examination in diagnosis of posterior pelvic ring injuries. J Orthop Trauma 17: 257-261

8. Pehle B, Nast-Kolb D, Oberbeck R et al. (2003) Wertigkeit der körperlichen und radiologischen $\mathrm{Ba}-$ sisdiagnostik des Beckens im Schockraum. Unfallchirurg 106: 642-648

9. Pohlemann $\mathrm{T}$, Tscherne $\mathrm{H}$, Baumgartel $\mathrm{F}$ et al (1996) Pelvic fractures: epidemiology, therapy and long-term outcome. Overview of the multicenter study of the Pelvis Study Group. Unfallchirurg 9: 160

10. Rommens PM, Vandershot PM, Broos PL (1992) Conventional radiography and $\mathrm{CT}$ examination of pelvic ring fractures. Unfallchirurg 95: 387-392

11. Ryan MD, Henderson JJ (1992) The epidemiology of fractures and fracture-dislocation of the cervical spine. Injury 23: 38-40

12. Schädel-Höpfner M, Celik I, Stiletto R et al. (2002) Häufigkeit hinterer Läsionen bei vorderen Beckenringfrakturen. Chirurg 1013: 1013-1018

13. White AA, Panjabi MM (1990) The problem of clinical instability of the human spine: a systematic approach. In: White AA, Panjabi MM (eds) Clinical biomechanics of the spine. Lippincott, Philadelphia, pp 277-378 